\title{
Directed Evolution of Artificial Metalloenzymes for In Vivo Metathesis
}

\author{
Markus Jeschek ${ }^{1}$, Raphael Reuter ${ }^{2}$, Tillmann Heinisch², Christian Trindler ${ }^{2}$, Juliane Klehr $^{2}$, \\ Sven Panke ${ }^{1 *}$, Thomas R. Ward ${ }^{2 *}$ \\ ${ }^{1}$ Department of Biosystems Science and Engineering, ETH Zurich, Basel, Switzerland. \\ ${ }^{2}$ Department of Chemistry, University of Basel, Basel CH-4056, Switzerland.
}

\begin{abstract}
Artificial metalloenzymes that result from incorporation of an abiotic cofactor within a protein scaffold offer the opportunity to complement the natural enzymes' repertoire and to be evolved genetically. With synthetic biology applications in mind, it would be highly desirable to exploit the versatility of such hybrid catalysts in vivo. This is currently challenging due to cofactor inactivation and the difficulty for the cofactor to specifically bind to the cellular protein scaffold. Here, we show that an artificial metalloenzyme for ring-closing metathesis, an archetypal transition metal-catalyzed reaction without a natural equivalent, can be assembled and evolved in the periplasm of Escherichia coli. For this purpose, E. coli whole cells harboring a periplasmic streptavidin were combined with a biotinylated Hoveyda-Grubbs metathesis catalyst in vivo. The biotin moiety serves as an affinity anchor to ensure selective and tight binding of the cofactor to streptavidin. Using a fluorogenic substrate surrogate, the performance of the resulting artificial metathase was improved by directed evolution, demonstrating the evolvability of such catalysts in vivo. The strain with the final quintuple mutant revealed a 5.4 -fold $( \pm 1.2$-fold) increased cell-specific activity which may be traced back to the increased flexibility of key residues in the active site pocket. The purified and evolved artificial metathase displayed over 600 turnovers for the water soluble 2,2diallylpropane-1,3-diol, outperforming both commercial catalysts and the wild-type metathase. This marks the starting point for novel metabolic schemes employing new-tonature metalloenzymes.
\end{abstract}

The field of biocatalysis has matured from harnessing natural enzymes to directed evolution of biological systems from single enzymes to complex organisms ${ }^{1}$. Recently, tools have emerged, to expand nature's enzymatic repertoire with abiotic reactions ${ }^{2,3}$. Artificial metalloenzymes (ArMs), which result from combining organometallic cofactors with proteins, offer an attractive means to engineer new-to-nature reactions. Three complementary

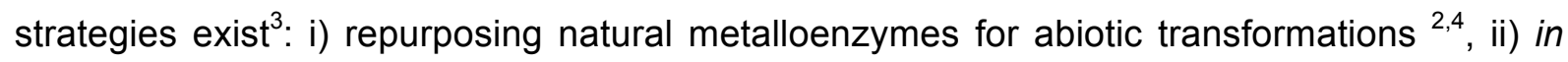
silico metalloenzyme (re-)design ${ }^{5-7}$ and iii) incorporation of abiotic cofactors into proteins ${ }^{8-11}$. The latter offers the opportunity to design a wide variety of ArMs for non-natural reactions. 
However, many metal cofactors are inhibited by cell components and thus require purification of the protein ${ }^{12-15}$. This limits the throughput of genetic optimization schemes applied to ArMs and their applicability in vivo to expand natural metabolism. Herein, we report on our efforts to compartmentalize and evolve in vivo an ArM for olefin metathesis (i.e. a metathase), which represents an archetypal organometallic reaction ${ }^{16-22}$ without equivalent in nature. Building upon the work of Song and Tezcan on an artificial metallohydrolase, we exploit the periplasm of $E$. coli as reaction compartment for the metathase since it offers an auspicious environment for ArMs mainly due to low concentrations of inhibitors such as glutathione $(\mathrm{GSH})$, which has recently been identified as a major inhibitor of $\mathrm{ArMs}^{15}$. This strategy facilitated the assembly of a functional metathase in vivo and its directed evolution with substantially increased throughput compared to conventional approaches relying on purified protein variants. The evolved metathase compares favorably with commercial catalysts, shows activity for different metathesis substrates and can be further evolved into different directions by adjusting the developed workflow. This represents the first systematic implementation and evolution of an ArM catalyzing an abiotic reaction in vivo with potential applications in non-natural metabolism and beyond.

In the past decade, the biotin-streptavidin technology has proven versatile for the generation of $\mathrm{ArMs}^{12,23-26}$. This may be traced back to the exquisite non-covalent affinity of biotinylated organometallic cofactors for streptavidin (SAV hereafter) ensuring their quantitative anchoring in an evolvable protein scaffold. To customize this system for in vivo applications, we designed a strain for periplasmic expression of streptavidin (SAV ${ }^{\text {peri }}$ hereafter) in E. coli. The resulting strain revealed good secretion of functional SAV into the periplasm with no detectable contamination in the cytoplasm (Fig. 1b).

Staining $E$. coli cells which harbor SAV ${ }^{\text {peri }}$ with Atto-565-biotin yielded a persisting strong fluorescent signal as analyzed by flow cytometric analysis whereas the dye-treated strain lacking $S A V^{\text {peri }}$ only exhibited low fluorescence (Fig. 1b). These experiments confirm the biotin-binding activity of SAV ${ }^{\text {peri }}$ and suggest that large hydrophobic biotinylated probes $\left(\mathrm{M}_{\mathrm{W}}(\right.$ Atto-565-biotin $\left.)=922 \mathrm{Da}\right)$ are taken-up into the periplasm.

Next, a biotinylated Hoveyda-Grubbs second generation catalyst (biot Ru hereafter) was selected to complete the holoenzyme (Fig. 1c) ${ }^{27}$. Prior experiments using purified SAV revealed that, upon incorporation within SAV, the biot $\sim \mathrm{Ru} \cdot \mathrm{SAV}$ complex catalyzes the ringclosing metathesis (RCM hereafter) of the umbelliferone precursor 1 to afford umbelliferone 2. Despite its poor performance as an RCM substrate in water ${ }^{27}$, we hypothesized that the formation of fluorescent umbelliferone $\mathbf{2}$, albeit in low yield, would offer an attractive means to monitor and evolve catalytic performance in vivo. Spiking an aqueous reaction mixture with $\mathrm{GSH}$ (10 equiv. vs. $50 \mu \mathrm{M}$ catalyst) lead to complete inhibition of 
biot Ru - SAV: no umbelliferone 2 was detected. In stark contrast, upon addition of GSSG instead of GSH (10 equiv. vs. $50 \mu \mathrm{M}$ catalyst), the metathesis activity of biot $\sim \mathbf{R u} \cdot \mathrm{SAV}$ was maintained (Extended Data Tab. 1). This suggests that the periplasm may indeed offer a suitable environment to harbor the artificial metathase as GSH is present in its oxidized GSSG form.

In order to confirm the assembly of the artificial metalloenzyme biot $\sim \mathbf{R u} \cdot \mathrm{SAV}$ within the periplasm, various E. coli strains were treated with the cofactor biot $\sim \mathbf{R u}$. Following incubation and washing, the ruthenium content was quantified by inductively coupled plasma optical emission spectrometry (ICP-OES, Fig. 1C). Although some unspecifically bound ruthenium is present in cells either without SAV at all (SAV ${ }^{-}$approx. $26{ }^{\prime} 000$ atoms/cell) or with cytoplasmic SAV (SAV ${ }^{\text {cyto }}$, approx. 31'000 atoms/cell), a threefold increase in ruthenium concentration was detected in the presence of SAV peri (approx. 80'000 atoms/cell), suggesting the transit of biot $\sim \mathbf{R u}$ through the outer membrane and its accumulation in SAV ${ }^{\text {peri }}$.

Next, the diolefin substrate 1 was added to $E$. coli cells bearing biot Ru $\cdot S A V^{\text {peri }}$. RCM activity yielding umbelliferone 2 was monitored by fluorescence (Fig. 1d). In contrast to the strain containing only the apoenzyme SAV ${ }^{\text {peri }}$, metathesis activity could be unambiguously detected only for $E$. coli cells containing the artificial metalloenzyme biot $\sim \mathbf{R u} \cdot \mathrm{SAV}^{\text {peri }}$ (Fig. $1 \mathrm{~d}$, photograph). Most importantly, quantification of the fluorescent signal revealed detectable metathesis activity for the strain containing biot $\sim \mathbf{R u} \cdot \mathrm{SAV} \mathrm{V}^{\text {peri }}$ whereas the free cofactor is largely inhibited in vivo in the absence of the SAV scaffold (Fig. 1d, bar chart). These experiments confirm: i) there is no E. coli-derived background ring-closing metathesis activity (i.e. bio-orthogonality); ii) the bare metathesis cofactor biot $\sim \mathbf{R u}$ is severely inhibited in E. coli; and iii) the periplasm is a propitious compartment to perform RCM with biot $\sim \mathbf{R u} \cdot S A V^{\text {peri }}$.

To the best of our knowledge, these results represent the first report of the assembly of an ArM in a whole cell setup and its subsequent use to catalyze an abiotic transformation in cellulo. By contrast, organometallic-mediated transformations performed so far in a cellular environment typically require multiple equivalents of the precious metal to achieve conversion ${ }^{28}$.

After demonstrating the assembly and activity of the metathase in the periplasm, we sought to evolve biot $\sim \mathbf{R u} \cdot \mathrm{SAV}^{\text {peri }}$ towards higher activity. We thus established a streamlined workflow for the growth and expression of different SAV ${ }^{\text {peri }}$ mutants in $E$. coli in a 96-well format (Fig. 2a). After the induction period, in which SAV ${ }^{\text {peri }}$ is synthesized and exported to the periplasm, the cells are harvested by centrifugation, resuspended in a buffer containing the cofactor and incubated for 30 minutes to allow for the assembly of the ArM in the periplasm. The buffer containing excess cofactor is then removed by centrifugation, the cells 
are resuspended in the reaction buffer and RCM is initiated by the addition of substrate 1 and monitored in a microtiter plate reader. This procedure allows for the screening of thousands of mutants in a matter of a few days and represents a substantial increase in throughput compared to the conventional workflow relying on purified mutants.

In order to improve the performance of the artificial metathase by directed evolution, we designed site saturation mutagenesis libraries of the twenty amino acid residues closest to the modeled position of ruthenium ${ }^{8}$ (Fig. 2b). Relying on degenerate NNK codons, analysis of 90 clones affords a $>94 \%$ probability to screen every possible amino acid at one position ${ }^{29}$. Fourteen of the twenty targeted positions revealed a potentially beneficial impact on catalysis in the fluorescence assay and were further selected for iterative saturation mutagenesis ${ }^{30}$. After evaluation of more than 3'000 clones, this directed evolution protocol led to the identification of the quintuple mutant $S V^{5 *}$ (biot Ru $\cdot S A V^{\text {peri }}$ V47A-N49K-T114Q-A119G$\mathrm{K} 121 \mathrm{R})$ that displayed a 5.4 -fold ( \pm 1.2 -fold) higher cell-specific activity compared to biot $\sim \mathbf{R u}$ - SAV ${ }^{\text {peri }}$ (Fig. 2c).

Next, the kinetic behavior of the free cofactor biot $\sim \mathbf{R u}$, biot $\sim \mathbf{R u} \cdot \mathrm{SAV}$ and biot $\sim \mathbf{R u} \cdot \mathrm{SAV}{ }^{5 *}$ was determined in vitro using purified protein samples and the resulting fluorescence of umbelliferone 2. Even though the limited substrate solubility prevented reaching the $V_{\max }$ domain, all three systems clearly display Michaelis-Menten behavior (Fig. 3a). As judged by the kinetic parameters derived from the best fit directed evolution led to an improvement both of the maximum reaction velocity $V_{\max }$ and the substrate affinity $K_{M}$. Biot $R \mathbf{R u} \cdot S A V^{5 *}$ displays a 2.0 -fold $\left( \pm 0.6\right.$-fold) improvement in catalytic efficiency $\left(k_{\text {cat }} / K_{M}\right)$ over the wild-type metathase. In order to verify the improved catalytic behavior, we tested the activity of Biot $\sim \mathrm{Ru} \cdot \mathrm{SAV}^{5 *}$ on three independently produced and purified protein batches (Extended Data Fig. 1) and varied the ratio between cofactor and SAV binding sites to ensure full complexation (Extended Data Fig. 2). Both experiments confirmed the aforementioned trend and the robustness of the proposed system.

The X-ray structure of biot $\mathbf{R u} \cdot \mathrm{SAV}^{5 *}$ (PDB 5F2B) reveals two conformations of the cofactor (I and II with $56 \%$ and $44 \%$ occupancy, Fig. 3b and Extended Data Fig. 3-4). Extensive steric clashes hamper the coexistence of two I conformers in adjacent cis-biotin binding-sites. However, both $\mathrm{I}-\mathrm{II}$ and II-II conformations are possible. The evolved metathase reveals greater biotin-vestibule loop flexibility when compared to the biot Ru - SAV structure (PDB 5IRA) as highlighted by analysis of the corresponding Bfactors (Extended Data Fig. 5). This may be traced back to three key mutations: $T 114 \mathrm{Q}$, A119G and V47A. Interestingly, these three mutations have the most pronounced positive effect during evolution (Fig. 2c), suggesting that increased loop-flexibility may be responsible for improved activity. 
Despite the versatility of the fluorescence assay which allowed kinetic activity monitoring in vivo, the substrate surrogate 1 is a notably poor RCM-substrate in water ${ }^{27}$ (Extended Data Tab. 2). Based on past experience with artificial metalloenzymes ${ }^{24}$, we anticipated that the evolved metathase may display broad substrate scope. We thus sought to determine whether the observed positive effect of the mutant on the substrate surrogate 1 would be reflected for typical RCM substrates. The in vitro catalytic performance for water-soluble substrates $\mathbf{3}$ and $\mathbf{5}$ was thus evaluated. For comparative purposes, two commercially available benchmark catalysts were included (i.e. second generation Hoveyda-Grubbs HGII and AquaMet AQM, Fig. 4a-b and Extended Data Fig. 6). For both substrates, the ArMs outperformed the free cofactor biot $\sim \mathbf{R u}$ as well as the two commercial catalysts. Interestingly, the evolved biot $\sim \mathbf{R u} \cdot \mathbf{S A V}^{5 *}$ was superior to biot $\sim \mathbf{R u} \cdot \mathrm{SAV}$ for 2,2-diallyl-1,3propanediol 3 (Fig. 4a). However, for the cationic diallyl-sulfonamide 5, biot Ru . SAV performed best (Fig. 4b). Inspection of the structure of biot Ru $\cdot S A V^{5 *}$ (Fig. 3b) revealed the proximity of the $121 \mathrm{R}$ residue to the Ru-alkylidene moiety. In view of the cationic nature of substrate $\mathbf{5}$, we speculated that the electrostatic repulsion with $121 \mathrm{R}$ may erode the performance of the ArM since it was evolved for the neutral substrate 1 . We therefore rerandomized position 121 by saturation mutagenesis and applied the periplasmic in vivo screening strategy using the cationic substrate 5 . The RCM activity to afford the dihydropyrrole 6 was quantified by UPLC-MS (see Methods section; Extended Data Fig. 7). This additional round of directed evolution led to the identification of a quintuple mutant (47A49K-114Q-119G-R121L, SAV ${ }^{5 * 2.0}$ hereafter) with improved activity for the charged substrate 5 both in vitro using purified proteins (Fig. 4b) as well as in vivo (Fig. 4c).

The proposed concept for the creation and evolution of an artificial metathase is thus applicable for various substrates and the evolved ArMs exhibit a certain degree of substrate specificity.

This work demonstrates the in vivo applicability of the streptavidin-biotin technology to create ArM catalyzing olefin metathesis, a reaction mechanism absent from the natural enzymes' repertoire. While the free cofactor biot $\sim \mathbf{R u}$ is nearly inactive in cellulo, the corresponding wild-type ArM biot $\sim \mathbf{R u} \cdot S A V^{\text {peri }}$ endows the cell with metathesis activity. The latter can be improved by directed evolution protocols for different substrates. We anticipate that this work will contribute to the future development and implementation of a set of bio-orthogonal tools to be utilized in new-to-nature metabolic pathways and beyond. 


\section{References}

1 Bornscheuer, U. T. et al. Engineering the third wave of biocatalysis. Nature 485, 185-194 (2012).

2 Renata, H., Wang, Z. J. \& Arnold, F. H. Expanding the enzyme universe: Accessing non-natural reactions by mechanism-guided directed evolution. Angew. Chem. Int. Ed. 54, 3351-3367 (2015).

3 Hyster, T. K., Ward, T. R. Genetic optimization of metalloenzymes: enhancing enzymes for non-natural reactions. Angew. Chem. Int. Ed. 55, $7344-7357$ (2016). doi: 10.1002/anie.201508816.

4 Coelho, P. S., Brustad, E. M., Kannan, A. \& Arnold, F. H. Olefinic cyclopropanation via carbene transfer catalzyed by engineered cytochrome P450 enzymes. Science 339, 307-310 (2013).

5 Khare, S. D. et al. Computational redesign of a mononuclear zinc metalloenzyme for organophosphate hydrolysis. Nature Chem. Biol. 8, 294-300 (2012).

6 Song, W. J. \& Tezcan, F. A. A designed supramolecular protein assembly with in vivo enzymatic activity. Science 346, 1525-1528 (2014).

7 Zastrow, M. L., Peacock, A. F. A., Stuckey, J. A. \& Pecoraro, V. L. Hydrolytic catalysis and structural stabilization in a designed metalloprotein. Nature Chem. 4, 118-123 (2012).

8 Creus, M. et al. X-ray structure and designed evolution of an artificial transfer hydrogenase. Angew. Chem. Int. Edit. 47, 1400-1404 (2008).

9 Lewis, J. C. Artificial metalloenzymes and metallopeptide catalysts for organic synthesis. ACS Catal. 3, 2954-2975 (2013).

10 Yu, F. T. et al. Protein design: Toward functional metalloenzymes. Chem. Rev. 114, 3495-3578 (2014).

11 Key, H.M., Dydio, P., Clark, D.S., Hartwig, J.F. Abiological catalysis by artificial haem proteins containing noble metals in place of iron. Nature. (2016). doi: 10.1038/nature17968.

12 Reetz, M. T., Peyralans, J. J. P., Maichele, A., Fu, Y. \& Maywald, M. Directed evolution of hybrid enzymes: Evolving enantioselectivity of an achiral Rh-complex anchored to a protein. Chem. Commun., 4318-4320 (2006).

13 Srivastava, P., Yang, H., Ellis-Guardiola, K. \& Lewis, J. C. Engineering a dirhodium artificial metalloenzyme for selective olefin cyclopropanation. Nature Commun. 6, 7789 (2015).

14 Sauer, D. F. et al. A highly active biohybrid catalyst for olefin metathesis in water: Impact of a hydrophobic cavity in a $\beta$-barrel protein. ACS Catal., 7519-7522 (2015).

15 Wilson, Y. M., Dürrenberger, M., Nogueira, E. S. \& Ward, T. R. Neutralizing the detrimental effect of glutathione on precious metal catalysts. J. Am. Chem. Soc. 136, 8928-8932 (2014).

16 Grubbs, R. H. Olefin-metathesis catalysts for the preparation of molecules and materials (Nobel lecture). Angew. Chem. Int. Ed. 45, 3760-3765 (2006).

17 Schrock, R. R. Multiple metal-carbon bonds for catalytic metathesis reactions (Nobel lecture). Angew. Chem. Int. Ed. 45, 3748-3759 (2006).

18 Chauvin, Y. Olefin metathesis: The early days (Nobel lecture). Angew. Chem. Int. Ed. 45, 37403747 (2006).

19 Hoveyda, A. H. \& Zhugralin, A. R. The remarkable metal-catalysed olefin metathesis reaction. Nature 450, 243-251 (2007). 
20 Fürstner, A. Teaching metathesis "simple" stereochemistry. Science 341, 1357 (2013).

21 Burtscher, D. \& Grela, K. Aqueous olefin metathesis. Angew. Chem. Int. Ed. 48, 442-454 (2009).

22 Lin, Y. A., Chalker, J. M., Floyd, N., Bernardes, G. J. L. \& Davis, B. G. Allyl sulfides are privileged substrates in aqueous cross-metathesis: Application to site-selective protein modification. $J$. Am. Chem. Soc. 130, 9642-9643 (2008).

23 Wilson, M. E. \& Whitesides, G. M. Conversion of a protein to a homogeneous asymmetric hydrogenation catalyst by site-specific modification with a diphosphinerhodium(I) moiety. J. Am. Chem. Soc. 100, 306-307 (1978).

24 Ward, T. R. Artificial metalloenzymes based on the biotin-avidin technology: Enantioselective catalysis and beyond. Acc. Chem. Res. 44, 47-57 (2011).

25 Ilie, A. \& Reetz, M. T. Directed evolution of artificial metalloenzymes. Isr. J. Chem. 55, 51-60 (2015).

26 Lo, C., Ringenberg, M. R., Gnandt, D., Wilson, Y. \& Ward, T. R. Artificial metalloenzymes for olefin metathesis based on the biotin-(strept)avidin technology. Chem. Commun. 47, 1206512067 (2011).

27 Kajetanowicz, A., Chatterjee, A., Reuter, R. \& Ward, T. R. Biotinylated metathesis catalysts: Synthesis and performance in ring closing metathesis. Catal. Lett. 144, 373-379 (2014).

28 Volker, T., Dempwolff, F., Graumann, P. L. \& Meggers, E. Progress towards bioorthogonal catalysis with organometallic compounds. Angew. Chem. Int. Ed. 53, 10536-10540 (2014).

29 Reetz, M. T., Kahakeaw, D. \& Lohmer, R. Addressing the numbers problem in directed evolution. Chembiochem 9, 1797-1804 (2008).

30 Reetz, M. T. \& Carballeira, J. D. Iterative saturation mutagenesis (ISM) for rapid directed evolution of functional enzymes. Nature Prot. 2, 891-903 (2007). 


\section{Methods}

\section{Chemicals and Reagents}

If not stated otherwise all chemicals and reagents were obtained from Sigma Aldrich (Buchs, Switzerland). Restriction enzymes were obtained from New England Biolabs (Ipswich, MA, USA). The AquaMet catalyst was purchased from Apeiron Synthesis S.A., Wroclaw, Poland.

\section{Cloning of SAV expression constructs}

All plasmids used in this study are collected in Supplementary Table 1. In order to construct the periplasmic expression vector for SAV, the gene for T7-tagged SAV was PCR-amplified from pET-11b-SAV ${ }^{31}$ using primers 1 and 2 (Suppl. Tab. 2) in order to add the 21 amino acid OmpA signal peptide (MKKTAIAIAVALAGFATVAQA) to the N-terminus of SAV. The PCR product was digested with restriction enzymes $\mathrm{Ndel}$ and $\mathrm{BamHI}$, gel-purified and ligated into the target vector $\mathrm{pET}-30 \mathrm{~b}(+)$ (Merck Millipore) pre-treated with the same enzymes. The resulting expression vector, designated $\mathrm{pET}-30 \mathrm{~b}-\mathrm{SAV}^{\text {peri }}$, carries the OmpA-SAV fusion gene under the control of a $\mathrm{P}_{\mathrm{T} 7}$ promoter. In order to construct a comparable cytoplasmic expression construct, the T7-tagged SAV gene from pET11b-SAV was PCR-amplified without adding additional amino acids using primers 3 and 2 (Suppl. Tab. 2) and subsequently cloned into $\mathrm{pET}-30 \mathrm{~b}(+)$ by restriction digest $(\mathrm{Ndel}$ and $\mathrm{BamHI})$ and ligation, resulting in plasmid $\mathrm{pET}-30 \mathrm{~b}-\mathrm{SAV} \mathrm{V}^{\text {cyto }}$.

\section{Construction of the periplasmic expression strain}

All strains used in this study are summarized in Supplementary Table 3. A strain for periplasmic expression was constructed that combines the ease of library generation with the compatibility with the T7-expression system. Therefore, the gene of the T7 RNA polymerase was integrated into the chromosome of E. coli TOP10 ( $\mathrm{F}^{-}$mcrA $\Delta$ (mrr-hsdRMS-mcrBC)

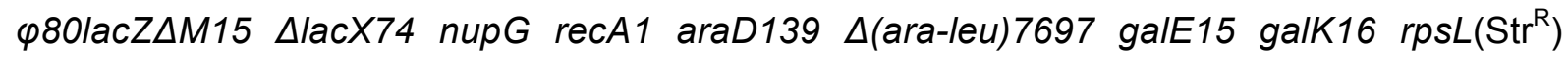
endA1 $\lambda^{-}$, Thermo Fisher Scientific) using the $\lambda D E 3$ Lysogenization Kit (Merck Millipore). The resulting lysogen was designated E. coli TOP10(DE3). 


\section{Shake flask cultivation and expression of SAV}

E. coli TOP10(DE3) containing the respective expression plasmid for SAV (pET-30b-SAV ${ }^{\text {peri }}$ or pET-30b-SAV $\left.{ }^{\text {cyto }}\right)$ was cultivated in of Luria-Bertani (LB) medium (50 ml) $)^{32}$ supplemented with kanamycin $\left(50 \mathrm{mg} \cdot \mathrm{I}^{-1}\right)$ in shake flasks $\left(500 \mathrm{ml}, 37^{\circ} \mathrm{C}, 200 \mathrm{rpm}\right)$. An LB pre-culture was diluted 1:100 in fresh medium and incubation was performed until an optical density at $600 \mathrm{~nm}\left(\mathrm{OD}_{600}\right)$ of $\sim 0.5$ was reached. Subsequently, the cultivation temperature was lowered to $20^{\circ} \mathrm{C}$, and the expression of SAV was induced by addition of isopropyl- $\beta$-Dthiogalactopyranoside (IPTG, $50 \mu \mathrm{M}$ ) and cells were harvested by centrifugation (6'000 rcf, 2 min) after four hours of induction. The cell pellet was either i) fractioned to analyze the cellular protein content or ii) subjected to flow-cytometry.

\section{Fractioning of cellular protein and analysis}

To separate the periplasmic and the cytoplasmic fraction of cellular proteins, the PeriPreps Periplasting Kit (Epicentre Technologies, Madison, WI, U.S.A.) was used. For in-gel-staining of SAV, biotin-4-fluorescein $(10 \mu \mathrm{M})$ was added to the respective fractions prior to SDSPAGE analysis. Due to the binding to the biotinylated fluorescent dye, SAV can be visualized under UV light in the gel (prior to staining with Coomassie Brilliant Blue). Please note that due to its high stability, SAV remains tetrameric and fully functional even under the otherwise denaturing conditions of SDS-PAGE.

\section{Flow-cytometry}

In order to stain SAV in whole cells of $E$. coli, the pellet of a shake flask expression culture was resuspended in phosphate buffer saline $\left(\mathrm{PBS}^{32}\right)$ to a final $\mathrm{OD}_{600}$ of 1 prior to the addition of Atto-565-biotin ( $2 \mu \mathrm{M}$, Atto-Tec $\mathrm{GmbH}$, Siegen, Germany). Cells were incubated on ice (30 $\mathrm{min}$ ) and subsequently subjected to three wash cycles by centrifugation (6'000 rcf, $2 \mathrm{~min}$ ) and resuspension of the pellet in PBS. The resulting cell suspensions were analyzed on a BD LSR Fortessa SORP (BD Biosciences, Allschwil, Switzerland) using a $561 \mathrm{~nm}$ laser for the excitation of Atto-565-biotin and a 610/20BP-600LP filter combination for analysis of the emitted fluorescence signal (peak area). The histograms displayed comprise data of 100'000 ungated events for each sample.

\section{Cultivation in 96-well plates}

For expression of SAV (and mutants thereof) in 96-deepwell format, LB medium (500 $\mu \mathrm{l}$ ) supplemented with kanamycin $\left(50 \mathrm{mg} \cdot \mathrm{I}^{-1}\right)$ were inoculated from a single colony and grown until stationary phase $\left(37^{\circ} \mathrm{C}, 300 \mathrm{rpm}, 50 \mathrm{~mm}\right.$ shaking amplitude). This pre-culture (aliquot of $30 \mu \mathrm{L})$ was used to inoculate a main culture $(1 \mathrm{ml})$ in a modified ZYM-5052 medium ${ }^{33}$ which 
lacked lactose as auto-induction agent but contained kanamycin $\left(50 \mathrm{mg} \cdot \mathrm{I}^{-1}\right)$. Induction was performed by the addition of IPTG $\left([\mathrm{IPTG}]_{\text {final }}=50 \mu \mathrm{M}\right)$ and continued for four hours before harvesting. Subsequently, a fraction of the cultures $(100 \mu \mathrm{l})$ was set aside for $\mathrm{OD}_{600}$ determination and the remaining suspension was subjected either to i) ICP-OES quantification or to ii) metathesis with whole cells.

\section{Metathesis activity in the presence of GSH or GSSG}

The activity of the ArM biot Ru - SAV was quantified in the reaction buffer (100 mM sodium acetate, $\mathrm{pH} 4.0,0.5 \mathrm{M} \mathrm{MgCl}_{2}, 2.5 \% \mathrm{DMSO}$ ) both in the presence and absence of 10 equivalents of glutathione (GSH or GSSG; $500 \mu \mathrm{M}$ ) relative to the amount of the cofactor biot $\sim \mathbf{R u}(50 \mu \mathrm{M})$. The reaction mixture (total volume: $200 \mu \mathrm{l}$ ) was incubated shaking (16 h, $37^{\circ} \mathrm{C}$ ) and after the reaction $800 \mu$ of methanol containing 2-phenylethanol (final conc.: $100 \mu \mathrm{M})$ as an internal standard were added. After centrifugation (5 min, 21'000 rcf, $4^{\circ} \mathrm{C}$ ) $500 \mu \mathrm{l}$ of the supernatant were diluted with $500 \mu \mathrm{l}$ de-ionized water and the samples were subjected to UPLC analysis.

UPLC analysis was performed using a Waters $\mathrm{H}$-Class Bio using a BEH C18 1,7 $\mu \mathrm{M}$ column and a flow rate $0.6 \mathrm{ml} \cdot \mathrm{min}^{-1}$ (eluent $A: 0.1 \%$ formic acid in water, eluent $\mathrm{B}: 0.1 \%$ formic acid in acetonitrile; gradient: 0 min: $90 \% A, 10 \%$ B; $0.5 \min : 90 \% A, 10 \% B ; 2.5 \min : 10 \% A, 90 \%$ B; $3.5 \mathrm{~min}: 90 \% \mathrm{~A}, 10 \% \mathrm{~B} ; 4.5 \mathrm{~min} 90 \% \mathrm{~A}, 10 \% \mathrm{~B}$ ). The UV signal at $210 \mathrm{~nm}$ was used for quantification and concentrations of the metathesis product umbelliferone 2 (retention time: $1.38 \mathrm{~min}$ ) were determined on the basis of a standard curve with commercially available umbelliferone (Sigma Aldrich, Switzerland).

\section{ICP-OES quantification of cellular ruthenium content}

In order to quantify the ruthenium content of cells, cultures from 96-deepwell plates were pelleted by centrifugation ( $3^{\prime} 220 \mathrm{rcf}, 12 \mathrm{~min}, 4^{\circ} \mathrm{C}$ ) and resuspended in ice-cold Tris/ $\mathrm{HCl}-$ buffer $(1 \mathrm{ml}, 50 \mathrm{mM}, \mathrm{pH} 7.4,0.9 \%(\mathrm{w} / \mathrm{v}) \mathrm{NaCl})$ containing biot $\mathbf{R u}(10 \mu \mathrm{M})$. After incubation on ice (30 $\mathrm{min}$ ), the cells were spun down (1'260 rcf, $8 \mathrm{~min}, 4^{\circ} \mathrm{C}$ ), the supernatant containing excess cofactor was discarded and the pellet was washed by resuspension in ice-cold Tris/HCl-buffer $(1 \mathrm{ml})$, centrifugation and careful removal of the supernatant. Afterwards, the pellet was resuspended in de-ionized water $(500 \mu \mathrm{l})$. Twelve replicates of this suspension were combined and concentrated nitric acid was added (550 $\mu, 65 \%)$ to fully digest the cellular material $\left(48 \mathrm{~h}, 110^{\circ} \mathrm{C}\right.$, pressure vials). The resulting clear solutions were diluted to a final volume of $10 \mathrm{ml}$ with de-ionized water (containing $1 \mathrm{ppm}$ yttrium as internal standard) and subjected to ICP-OES quantification. 
The obtained ppb-values $\left(1 \mathrm{ppb}=1 \mu \mathrm{g} \cdot \mathrm{I}^{-1}\right)$ for ruthenium were transformed into a molar concentration and the average ruthenium atom count per cell was calculated from the $\mathrm{OD}_{600}$ of the cultures prior to decomposition assuming an $\mathrm{OD}_{600}$-to-cell-number-correlation for $E$. coli in complex medium of $7.8 \cdot 10^{8} \mathrm{ml}^{-1} \cdot \mathrm{OD}_{600}{ }^{-1}$ as described by Volkmer and Heinemann ${ }^{34}$.

\section{Fluorescent metathesis assay with whole cells}

The cellular metathesis activity was quantified using a fluorescent assay. For this purpose, cell cultures from 96-deepwell plate cultivations (see above) were pelleted by centrifugation (3'220 rcf, $12 \mathrm{~min}$ ) and resuspended in ice-cold Tris/HCl-buffer $(500 \mu \mathrm{l}, 50 \mathrm{mM}, \mathrm{pH} 7.4$ ) containing biot $\sim \mathrm{Ru}(2.1 \mu \mathrm{M})$. This buffer was supplemented with $\mathrm{NaCl}(0.9 \%(\mathrm{w} / \mathrm{v}))$ to adjust to a physiological $\mathrm{NaCl}$ concentration. To allow cellular uptake of biot $\sim \mathbf{R u}$, the suspensions were incubated on ice for 30 minutes, spun down (1'260 rcf, $8 \mathrm{~min}$ ) and the supernatant containing excess cofactor was discarded. The pellets were resuspended in the reaction buffer $\left(160 \mu \mathrm{l}, 100 \mathrm{mM}\right.$ sodium acetate, $\mathrm{pH} 4.0,0.5 \mathrm{M} \mathrm{MgCl}_{2}$ ) and the reaction was initiated by addition of these cell suspensions $(150 \mu \mathrm{l})$ to the substrate solution $(50 \mu \mathrm{l}$ reaction buffer containing $40 \mathrm{mM}$ precursor 1 and $20 \%$ DMSO) leading to a final substrate concentration of $10 \mathrm{mM}$ and $5 \%$ DMSO. As the metathesis product umbelliferone 2 is fluorescent, the reaction progress was monitored by fluorescence in a microtiter plate reader $\left(\lambda_{\mathrm{Ex}}=322 \pm 4.5 \mathrm{~nm}\right.$, $\lambda_{E m}=440 \pm 10 \mathrm{~nm}$; Infinite M1000 PRO, Tecan Group AG, Männedorf, Switzerland) at $37^{\circ} \mathrm{C}$ and agitation ( $6 \mathrm{~mm}$ amplitude, orbital). Cell specific metathesis activity is specified as the slope of the increasing fluorescence signal in the linear range of the reaction normalized by the $\mathrm{OD}_{600}$ of the respective culture.

\section{Site-saturation mutagenesis of SAV and screening}

To generate diversity in the scaffold protein SAV a focused, semi-rational strategy was pursued: the 20 amino acid residues closest to the ruthenium ion (see Suppl. Tab. 4) in a related ArM structure ${ }^{8}$ were selected and individually randomized in SAV ${ }^{\text {peri }}$ by site saturation mutagenesis using NNK codons $^{30}$. Degeneration was introduced by application of the Quikchange Site Directed Mutagenesis Protocol (Stratagene) using degenerate oligos (see Suppl. Tab. 4) and the transformed libraries were checked for diversity by Sangersequencing of at least four individual clones prior to screening. To evaluate the performance of the SAV $\mathrm{V}^{\text {pri }}$-variants, the aforementioned fluorescent metathesis assay was carried out with at least 90 clones from the library in one 96-well plate (three replicates of "wildtype" SAV (parent) and three replicates of an empty vector control (pET-30b(+), lacking SAV ${ }^{\text {peri }}$ ) were included as controls). Evaluating 90 members of an NNK-library ensures a greater than $94 \%$ likelihood of screening all twenty amino acid residues in the respective position ${ }^{29}$. To compensate for biological variance, promising clones were isolated and subjected to a 
replicate assay which was identical to the protocol described above but using eight independent cultures per clone.

After this first screening round, promising residues were ordered according to their potential impact on catalysis and mutations were combined using iterative saturation mutagenesis $(\mathrm{ISM})^{30}$.

\section{Enzyme Kinetics}

To perform kinetic experiments on the artificial metathase, the initially used T7-tagged SAV as well as the quintuple mutant SAV ${ }^{5 *}$ (V47A-N49K-T114Q-A119G-K121R) isolated after ISM were cloned into a cytoplasmic expression vector (see section "Cloning of SAV expression constructs") and purified on an iminobiotin sepharose column as described elsewhere ${ }^{35}$. The biotin binding capacity was determined using a fluorescent quenching assay $^{36}$. Kinetic measurements were performed in reaction buffer $(200 \mu \mathrm{l}$ total volume, 100 $\mathrm{mM}$ sodium acetate, $\mathrm{pH} 4.0,0.5 \mathrm{M} \mathrm{MgCl}_{2}, 11.5 \%$ ( $\left.\mathrm{v} / \mathrm{v}\right) \mathrm{DMSO}$ ) containing biot $\mathbf{R u}(50 \mu \mathrm{M})$ both in the presence and absence of purified SAV (100 $\mu \mathrm{M}$ binding sites of either T7-tagged SAV or the quintuple mutant) as well as the substrate 1 (variable concentrations, 0-5 mM) for fluorescent RCM. The reaction was monitored in a microtiter plate reader as described in section "Fluorescent metathesis assay with whole cells". The maximum velocity of the reaction was determined from the fluorescent signal curve by linear regression. In order to retrieve kinetic parameters the reaction velocities were plotted over the respective substrate concentrations using the software GraphPad Prism (GraphPad Software Inc., version 6.05) and applying the integrated "Michaelis-Menten" least squares fit with no constraints for the maximum velocity $v_{\max }$ and $K_{\mathrm{m}}$.

\section{Protein Crystallization and Catalyst Soaking}

Crystals of SAV and variant $\mathrm{SAV}^{5 *}$ were obtained at $20^{\circ} \mathrm{C}$ within two days by the sitting-drop vapor diffusion technique mixing $1 \mu \mathrm{l}$ crystallization buffer $(1.5 \mathrm{M}$ ammonium sulfate, $0.1 \mathrm{M}$ sodium acetate, $\mathrm{pH} 4.0$ ) and $4 \mu \mathrm{l}$ protein solution $\left(26 \mathrm{mg} \cdot \mathrm{ml}^{-1}\right.$ lyophilized protein in water). The droplet was equilibrated against a reservoir solution of $100 \mu$ l crystallization buffer. Subsequently, single crystals were soaked for two days at $20{ }^{\circ} \mathrm{C}$ in a soaking buffer, which was prepared by mixing $1 \mu \mathrm{l}$ of a $10 \mathrm{mM}$ stock solution of complex biot $\mathbf{R u}$ (in $50 \%$ aqueous DMSO) and $9 \mu$ crystallization buffer. After the soaking, crystals were transferred for 30 seconds into a cryo-protectant solution consisting of $25 \%(\mathrm{v} / \mathrm{v})$ glycerol in crystallization buffer. Next, crystals were shock-frozen in liquid nitrogen. Additional soaking of the above metathase crystals with substrate surrogate 1 did not lead to fluorescence. We thus conclude that the multiple catalytic steps (e.g. ligand displacement, cross-metathesis etc.) required to ultimately liberate umbelliferone 2 cannot take place within a crystal. 


\section{Data Processing and Structure Refinement}

X-ray diffraction data were collected at the Swiss Light Source beam line X06DA at a wavelength of $1 \AA$ and processed with software $X^{2} S^{37}$ and AIMLESS (CCP4 Suite) $)^{38}$. The structure was solved by molecular replacement using program PHASER (CCP4 Suite) ${ }^{38}$ and the structure $2 \mathrm{QCB}$ from the PDB as input model with ligand and water molecules removed. For structure refinement REFMAC5 (CCP4 Suite) ${ }^{39}$ and PHENIX.REFINE ${ }^{40}$ were used. Ligand manipulation was carried out with program REEL using the small molecule crystal structure ABEJUM from the Cambridge Structural Database as input model ${ }^{40}$. For water picking and electron density and structure visualization, the software $\mathrm{COOT}^{41}$ was used. Figures were drawn with PyMOL (the PyMOL Molecular Graphics System, Version 1.5.0.5, Schrödinger, LLC). Crystallographic details, processing and refinement statistics are given in Supplementary Table 5.

\section{Crystal Structure of biot $\sim \mathrm{Ru} \cdot \mathrm{SAV}^{5 *}$}

There is one streptavidin monomer in the asymmetric unit from which a tetramer can be generated by application of two orthogonal crystallographic 2-fold symmetry axes. The $12 \mathrm{~N}$ terminal residues of the 77 -tag and 25 residues at the $\mathrm{C}$-terminus are not resolved, probably due to disorder.

Residual electron density in the biotin-binding site and the biotin vestibule as well as two strong anomalous dispersion density peaks in the biotin vestibule (Extended Data Fig. 3) suggested modeling of complex biot Ru in two conformations I and II (56\% and $44 \%$ occupancy, respectively). This projects the Ru atom in either one of the two densities, in close proximity to a crystallographic 2-fold symmetry axis (Fig. 3b, Extended Data Fig. 3, Extended Data Fig. 4c-d). Only partial or no electron density was present for the mesityl linker and the terminal mesityl group, probably due to high flexibility. In conformer I, the lengthy dimesitylimidazolidine-Ru head group (DMI-Ru hereafter) reaches into the neighboring cis-related SAV monomer. The I conformer is stabilized mostly by hydrophobic interactions between the distal face of the DMI ligand and amino acid side chains within two

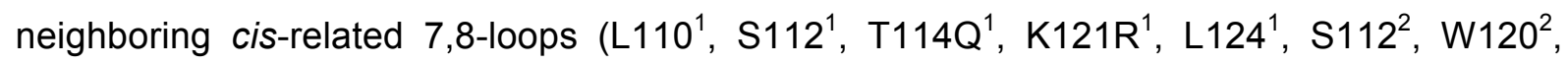
$\mathrm{K} 121 \mathrm{R}^{2}, \mathrm{~L} 124^{2}$ (superscripts refer to monomers 1 and 2 of the tetrameric SAV, respectively, Extended Data Fig. 4c). Besides the DMI ligand, two chloride ions could be modeled binding to the $\mathrm{Ru}$, but no density was found for the alkylidene, presumably due to high flexibility and/or low occupancy. The orientation of the chlorides was very similar to that in a small molecule crystal structure of the Grubbs-Hoyveda II-generation catalyst (ABEJUM from the Cambridge Structural Database) placing them nearly in trans position to each other. The Ru is largely solvent-exposed which could facilitate substrate binding and product release. In 
Figure $3 b$ the alkylidene was modeled binding to the ruthenium (magenta stick model) to highlight its orientation in the biotin vestibule. Conformer II is different from I by a rotation $\left(\sim 60^{\circ}\right)$ of the DMI-Ru moiety around an axis parallel to the cylinder axis of the SAV $\beta$-barrel (Fig. 3b, Extended Data Fig. 4d). This places the hydrophobic distal side of the DMI-ligand in proximity to amino acid side chains within loop-5, $6^{1}$ (A86, H87) and loop-7, $8^{1}$ (S112, T114Q) and loop $-4,5^{3}$ (D67, S69, A65) of a neighboring trans-related SAV monomer. Atom N49K-N $\varepsilon$ is located in close proximity to $\mathrm{Ru}(2.4 \AA)$. No electron density was found to model chloride ions and the alkylidene ligand bound to the Ru. Since the cofactor is bound in close proximity to a 2-fold crystal symmetry axis, formation of a cis-symmetry related neighboring cofactor by application of the crystal symmetry operation results in extensive steric clashes between the two cofactors in the orientation I. In contrast, coexistence of cofactor pairs either I-I or II-II orientation is sterically accessible (Extended Data Fig. 3a,c and e).

Normalized B-factors of residues within cofactor-flanking loop-7,8 are increased by up to $\sim 0.5$ when compared to related streptavidin structures that crystalized in the same space group and in a very similar unit cell (Extended Data Fig. 5b). This suggests increased loop7,8 flexibility (Fig. 3b, Extended Data Fig. 4c-d). This is likely to be caused by three factors: i) mutation $\mathrm{T} 114 \mathrm{Q}$ cleaves an $\mathrm{H}$-bond between threonine-OYH and T115-carbonyl oxygen (green dashed line in Fig. 3b and Extended Data Fig. 4a-b) and leads to a new H-bond between $\mathrm{T} 114 \mathrm{Q}-$ glutamine- $\mathrm{N} \varepsilon$ and $\mathrm{S} 112-\mathrm{O} Y \mathrm{H}$ (red dashed line in Figure $3 \mathrm{~b}$ and Extended Data Fig. 4c-d); ii) mutation A119G in the loop leads to increased entropy and iii) mutation $\mathrm{V} 47 \mathrm{~A}$ reduces steric hindrance between $\mathrm{V} 47 \mathrm{~A}$ in loop- $3,4^{2}$ and $\mathrm{W} 120$ in loop-7, $8^{1}$ (Fig. 3b and Extended Data Fig. 4c-d).

\section{Crystal Structure of biot Ru $\cdot$ SAV}

The overall structure of complex biot $\sim \mathbf{R u} \cdot \mathrm{SAV}$ is virtually identical to that of complex biot $\sim \operatorname{Ru} \cdot \mathrm{SAV}^{5 *}(\mathrm{rmsd}=0.25 \AA)$. As in the mutant, two strong residual electron density (FoFc cofactor omit map: $12 \sigma$ (conformer I) and $11 \sigma$ (conformer II), respectively) and anomalous dispersion density $(9 \sigma$ (conformer I) and $5 \sigma$ (conformer II), respectively) peaks were located within the biotin vestibule of a SAV monomer at the interface between two symmetry-related SAV monomers (Extended Data Fig. 3b,d and f). The same two cofactor conformations I and II found in mutant $S A V^{5 *}$ were modeled in the biotin binding vestibule of SAV with an occupancy of $50 \%$ for each conformer I and II (Extended Data Fig. 4a-b).

Strikingly, the side chain of residue L110 adopts two conformations with $50 \%$ occupancy each (Extended Data Fig. 4a-b). The close proximity of the terminal methyl group in L110 conformation $A$ to the aromatic mesityl ring $\left(\mathrm{L} 110-\mathrm{C} \delta \mathrm{H}---\right.$ mesityl $\left.\mathrm{I}_{\text {centroid }}=4.4 \AA\right)$ of cofactor conformation I suggests a stabilizing $\sigma^{\cdots} \cdots \pi$ interactions (Extended Data Fig. $4 a$, red star). The L110 side chain conformation B can only coexist with cofactor conformation II (Extended 
Data Fig. 4b). This hypothesis is supported by the fact that the same L110 side chain conformation $A$ is found in complex $\left[\left(C p^{*}\right) \operatorname{lr}(\right.$ Biot- $p$-L)Cl]-SAV-S112A (PDB 3PK2) which has an aromatic ring located in the same position as the mesityl linker in structure biot $\sim$ Ru $\cdot S A V$ suggesting a similar $\sigma^{\cdots} \pi$ interaction. In contrast, the side chain of L110 in apo-SAV (PDB 2BC3) adopts conformation B. In complex biot Ru - SAV, a water molecule is bound in proximity to L110 with $50 \%$ occupancy (Extended Data Fig. 4b). Steric clashes with L110 side chain in conformation $\mathrm{A}$ and the $\mathrm{NHC}$ ligand of biot $\sim \mathrm{Ru}$ conformer I suggests the water to be only present with L110 conformation B and biot Ru conformer II. Additionally, the side chain of L124 adopts two conformations with each $50 \%$ occupancy. Only conformation $\mathrm{L} 124^{\mathrm{A}}$ does not undergo steric clashes with a methyl group of the bridging mesityl moiety of cofactor conformer I (Extended Data Fig. 4a-b). Together, the conformational side chain flexibility of residues L110 and L124 reflects the presence of the two cofactor conformations I and II.

In contrast to ArM biot $\sim \mathrm{Ru} \cdot \mathrm{SAV}^{5 *}$ the normalized B-factors of residues within the cofactorflanking loop-7,8 in complex biot Ru . SAV do not show increased values when compared to those in related crystal structures (Extended Data Fig. 5b). Indeed, an H-bond is formed between T114-OYH and T115-carbonyl oxygen in complex biot $\mathbf{R u} \cdot \mathrm{SAV}$ that could rigidify the loop (Extended Data Fig. 4a-b).

\section{In vitro metathesis assay for substrate 3 (2,2-diallyl-1,3-propanediol)}

The conversion for the product 4 was quantified by ${ }^{1} \mathrm{H}-\mathrm{NMR}$. For this purpose, a deuterated reaction buffer was prepared from acetic acid- $\mathrm{d}_{4}$, dry $\mathrm{MgCl}_{2}$ and $\mathrm{D}_{2} \mathrm{O}$ with the same concentrations as for the reaction buffer used for substrate 1 (100 $\mathrm{mM}$ acetate, $0.5 \mathrm{M} \mathrm{MgCl}_{2}$ ). The $\mathrm{pH}$ was adjusted to 3.6 by addition of $1 \mathrm{M} \mathrm{NaOD}$ in $\mathrm{D}_{2} \mathrm{O}$ (with respect to $\mathrm{pD}=\mathrm{pH}+0.4$ ). For the reaction, $300 \mu \mathrm{l}$ of a substrate 3 stock solution (100 mM in deuterated reaction buffer) was mixed with $291 \mu \mathrm{L}$ of either a SAV solution (200 $\mu \mathrm{M}$ SAV binding sites in deuterated reaction buffer) or plain deuterated reaction buffer (for samples without SAV). Afterwards, 9 $\mu \mathrm{l}$ of a biot Ru (or HGII/AQM) stock solution (3.34 mM in DMSO- $\mathrm{d}_{6}$ ) was added to obtain a final concentration of $50 \mu \mathrm{M}$ and the reaction was performed for 16 hours at $37^{\circ} \mathrm{C}$ and 200 rpm. The mixture was analyzed by ${ }^{1} \mathrm{H}-\mathrm{NMR}$ and the yield of the reaction product 4 was quantified by comparing integrals (I hereafter) of the product 4 peaks at $3.41 \mathrm{ppm}$ and $2.05 \mathrm{ppm}$ and the substrate 3 peaks at $3.33 \mathrm{ppm}$ and $1.91 \mathrm{ppm}$ using the formula: Yield = $I_{\text {product }} /\left(I_{\text {product }}+I_{\text {substrate }}\right)$.

\section{In vitro metathesis assay for substrate $\mathbf{5}$ (diallyl sulfonamide)}

To quantify the conversion of substrate 5 , a $97 \mu$ aliquot of either SAV solution (200 $\mu$ M SAV binding sites in reaction buffer) or plain reaction buffer (for samples without SAV) were mixed 
with $100 \mu \mathrm{l}$ of a stock solution of substrate 5 ( $20 \mathrm{mM}$ in reaction buffer). Subsequently, $3 \mu \mathrm{l}$ of the respective catalyst/cofactor stock solution (3.34 mM in DMSO) were added to obtain a final concentration of $50 \mu \mathrm{M}$. The reaction was performed for 16 hours at $37^{\circ} \mathrm{C}$ and $200 \mathrm{rpm}$. Then, an aqueous solution of benzyltriethylammonium chloride $(100 \mu \mathrm{l}, 10 \mathrm{mM})$ was added as an internal standard and $700 \mu \mathrm{l}$ of methanol were added. The mixture was cleared by centrifugation and $250 \mu \mathrm{l}$ of the supernatant were mixed with $750 \mu \mathrm{l}$ of water for the final quantification of product 6 by UPLC-MS.

For the kinetic experiment, the reaction mixture was scaled up to a total volume of $1 \mathrm{ml}$ and $50 \mu \mathrm{l}$ aliquots of this mixture were collected at different time points of time and immediately injected into $950 \mu \mathrm{L}$ of a quenching solution $(0.5 \mathrm{mM}$ potassium cyanoacetate, $0.25 \mathrm{mM}$ benzyltriethyl-ammonium chloride (internal standard) in 50\% aqueous methanol). After removal of precipitated protein by centrifugation, the supernatant was analyzed by UPLCMS.

\section{Metathesis assay with whole cells and diallyl sulfonamide 5}

In order to quantify the cellular metathesis activity for substrate $\mathbf{5}$, a protocol analogous to that applied for the umbelliferone precursor $\mathbf{1}$ was applied. The substrate $\mathbf{5}$ (final concentration $10 \mathrm{mM}$ ) was added to whole cells and the samples were incubated at $37^{\circ} \mathrm{C}$ and 300 rpm for 16 hours. In order to quantify the conversion for the non-fluorescent product 6, an extraction was performed: $800 \mu \mathrm{l}$ of methanol were added to each sample and an extraction was carried out (one hour with vigorous shaking, $800 \mathrm{rpm}$ at room temperature). The samples were cleared by centrifugation, the supernatant was diluted with water (factor four) and analyzed by UPLC-MS using a calibration curve recorded for product 6 .

\section{Methods References}

31 Gallizia, A. et al. Production of a soluble and functional recombinant streptavidin in Escherichia coli. Prot. Expr. Purif. 14, 192-196 (1998).

32 Sambrook, J. F. \& Russell, D. W. Molecular Cloning : A Laboratory Manual. Cold Spring Harbor Laboratory, 3rd edition (2001).

33 Studier, F. W. Protein production by auto-induction in high-density shaking cultures. Prot. Expr. Purif. 41, 207-234 (2005).

34 Volkmer, B. \& Heinemann, M. Condition-dependent cell volume and concentration of Escherichia coli to facilitate data conversion for systems biology modeling. PLoS One 6, e23126 (2011).

35 Humbert, N., Schurmann, P., Zocchi, A., Neuhaus, J. M. \& Ward, T. R. High-yield production and purification of recombinant T7-tag mature streptavidin in glucose-stressed E. coli. Meth. Mol. Biol. 418, 101-110 (2008). 
36 Kada, G., Falk, H. \& Gruber, H. J. Accurate measurement of avidin and streptavidin in crude biofluids with a new, optimized biotin-fluorescein conjugate. Biochim. Biophys. Acta 1427, 33-43 (1999).

37 Kabsch, W. XDS. Acta Crystallogr. Sect. D Biol. Crystallogr. 66, 125-132 (2010).

38 Evans, P. R. An introduction to data reduction: Space-group determination, scaling and intensity statistics. Acta Crystallogr. Sect. D Biol. Crystallogr. 67, 282-292 (2011).

39 Murshudov, G. N., Vagin, A. A. \& Dodson, E. J. Refinement of macromolecular structures by the maximum-likelihood method. Acta Crystallogr. Sect. D Biol. Crystallogr. 53, 240-255 (1997).

40 Adams, P. D. et al. PHENIX: a comprehensive Python-based system for macromolecular structure solution. Acta Crystallogr. Sect. D Biol. Crystallogr. 66, 213-221 (2010).

41 Emsley, P. \& Cowtan, K. Coot: model-building tools for molecular graphics. Acta Crystallogr. Sect. D Biol. Crystallogr. 60, 2126-2132 (2004).

\section{End Notes}

\section{Acknowledgement}

The authors thank Dr. Philippe Marlière for enlightening discussions. This work was supported by funding from the European Commission Seventh Framework Programme [289572-METACODE] and the Swiss National Science Foundation as part of the NCCR Molecular Systems Engineering. The authors thank Dr. Mark Dessing and the single-cell facility (D-BSSE, ETH Zurich) for assistance with flow cytometry.

\section{Author contributions}

T.R.W. and S.P. conceived the project. M.J. developed the periplasmic screening platform and performed in vivo and directed evolution experiments. R.R. synthesized the cofactor and substrates. M.J. and R.R. performed the in vitro experiments. T.H. conducted the crystallography studies. C.T. performed ICP-OES and J.K. expressed and purified protein variants. T.R.W. and S.P. supervised the project. M.J., T.R.W. and S.P. wrote the manuscript.

\section{Author information}

The X-ray structures of the artificial metathases have been deposited in the Protein Data Bank (PDB) under accession numbers 5F2B and 5IRA.

Reprints and permissions information is available at www.nature.com/reprints.

Correspondence and requests for materials should be addressed to thomas.ward@unibas.ch. 


\section{Competing financial interests}

The authors declare no competing financial interests.

\section{Figures}

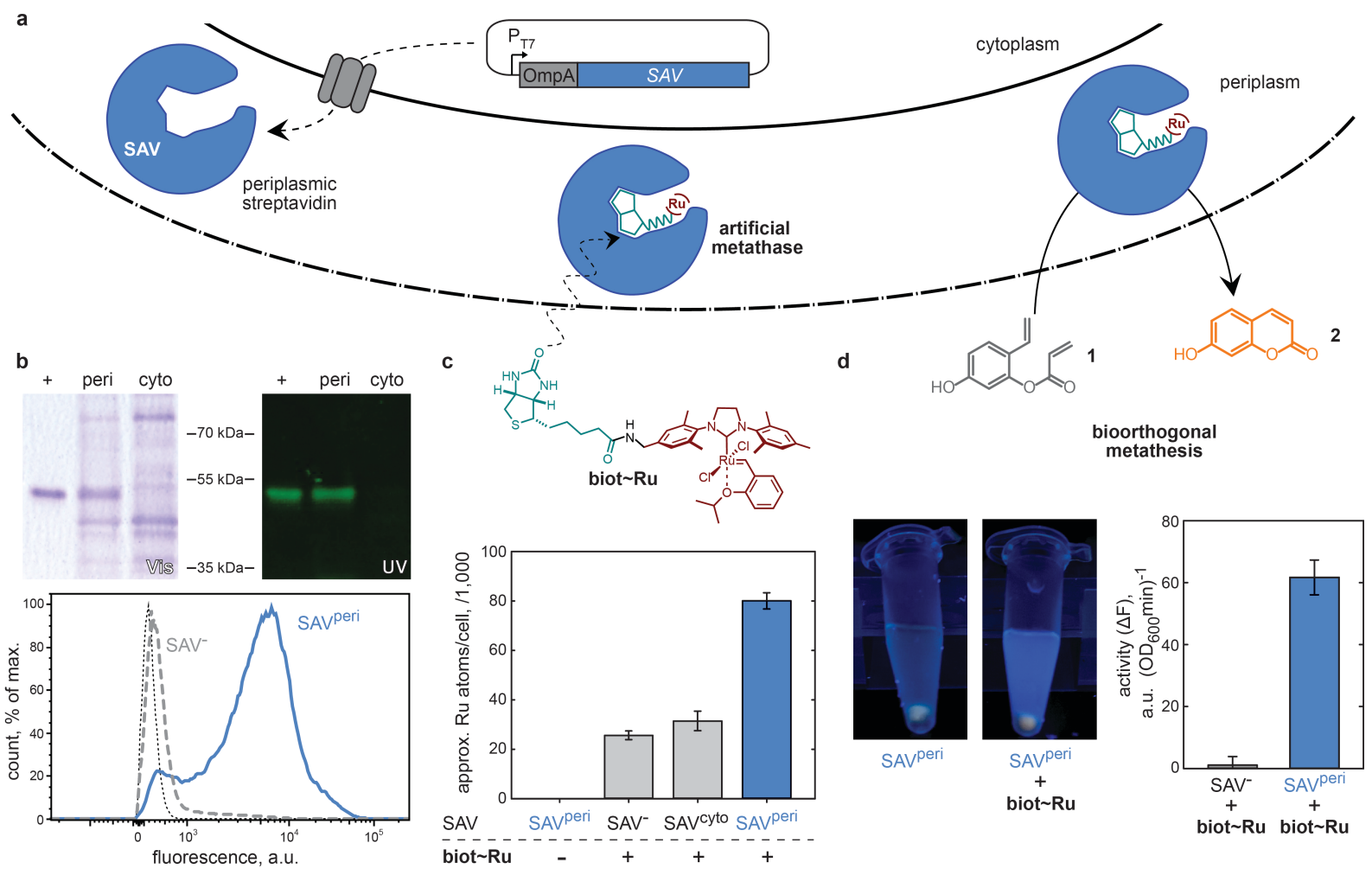

Figure 1 | Artificial Metalloenzymes for in vivo metathesis. a, Streptavidin (SAV) is secreted to the periplasm by fusion to an $\mathrm{N}$-terminal signal peptide (OmpA). b, Upper: OmpA fusion enables efficient secretion of SAV which accumulates in the periplasmic protein fraction ("peri") without contamination in the cytoplasm ("cyto"). Biotin binding is confirmed 
with a biotinylated fluorophore (UV). As positive control purified SAV (+) was included. Lower: Flow cytometry analysis by in vivo staining with a biotinylated fluorescent dye. Cells expressing SAV ${ }^{\text {peri }}$ remain highly fluorescent after washing whereas controls without SAV $\left(S A V^{-}\right)$and with $S A V^{\text {peri }}$ but without dye treatment (dotted line) are non-fluorescent. c, An artificial metathesis cofactor (biot $\sim \mathbf{R u}$ ) composed of a biotin anchor and a catalytic moiety is added to cells expressing SAV ${ }^{\text {peri }}$ thus creating a metathase. Cofactor uptake as determined by ICP-OES quantification of ruthenium is significantly higher for $S A V^{\text {peri }}$ as for $\mathrm{SAV}^{-}$or cytoplasmic SAV (SAV ${ }^{\text {cyto }}$ ). d, The metathase converts the non-fluorescent substrate 1 into fluorescent umbelliferone 2 by ring-closing metathesis (RCM). Cells with SAV ${ }^{\text {peri }}$ develop a signal due to the freely diffusible product 2 in the supernatant (left) which can be quantified (right) with a low background for $\mathrm{SAV}^{-}$. Bars are mean values for two (c)/three (d) independent replicate cultures with s.d..
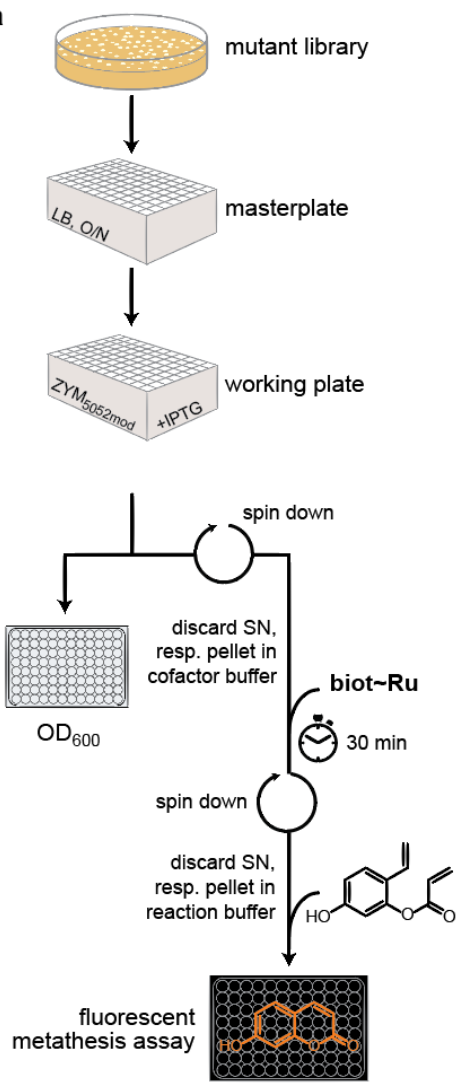

b
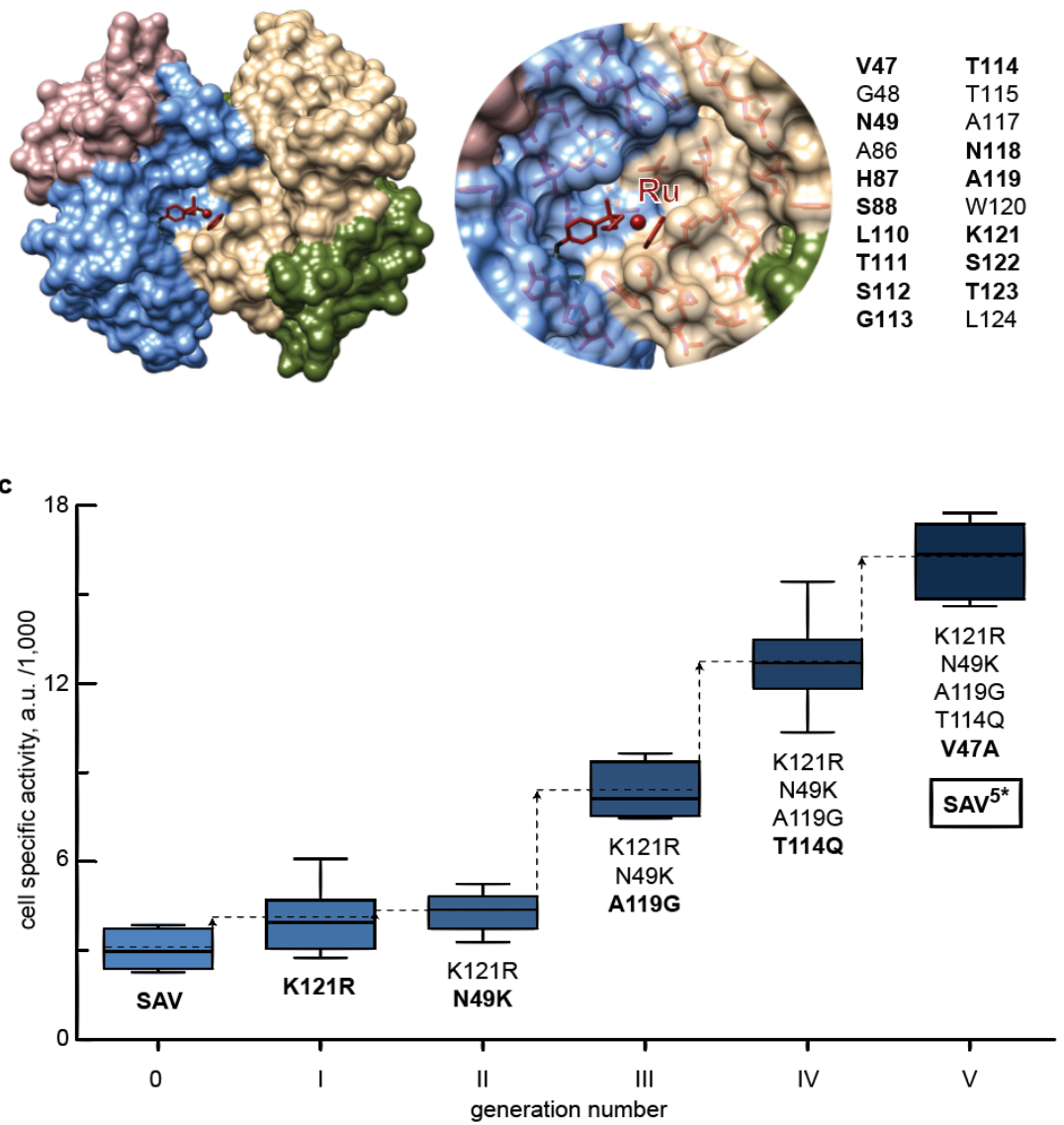

Figure 2 | Directed evolution of an artificial metathase. a, A screening workflow (96-well) was developed that allows for expression of SAV mutants and subsequent evaluation for their metathesis activity based on a whole-cell fluorescent assay. To increase the throughput, the substrate surrogate 1 was selected, thus allowing kinetic monitoring in vivo. b, The 20 
closest residues to the catalytic $\mathrm{Ru}$ ion in $\mathrm{SAV}^{8}$ were randomized by saturation mutagenesis (NNK codons) of which 14 revealed a positive impact (bold) on metathase activity. c, Iterative saturation mutagenesis ${ }^{30}$ on these 14 residues led to an optimized metathase bearing five mutations designated $\mathrm{SAV}^{5 *}$. Data from independent replicate cultures $(\mathrm{n}=8)$ are represented by boxes (median and first/third quartile) with whiskers (minimum/maximum of data). The horizontal dashed lines represent the mean cell specific activity of each generation. 

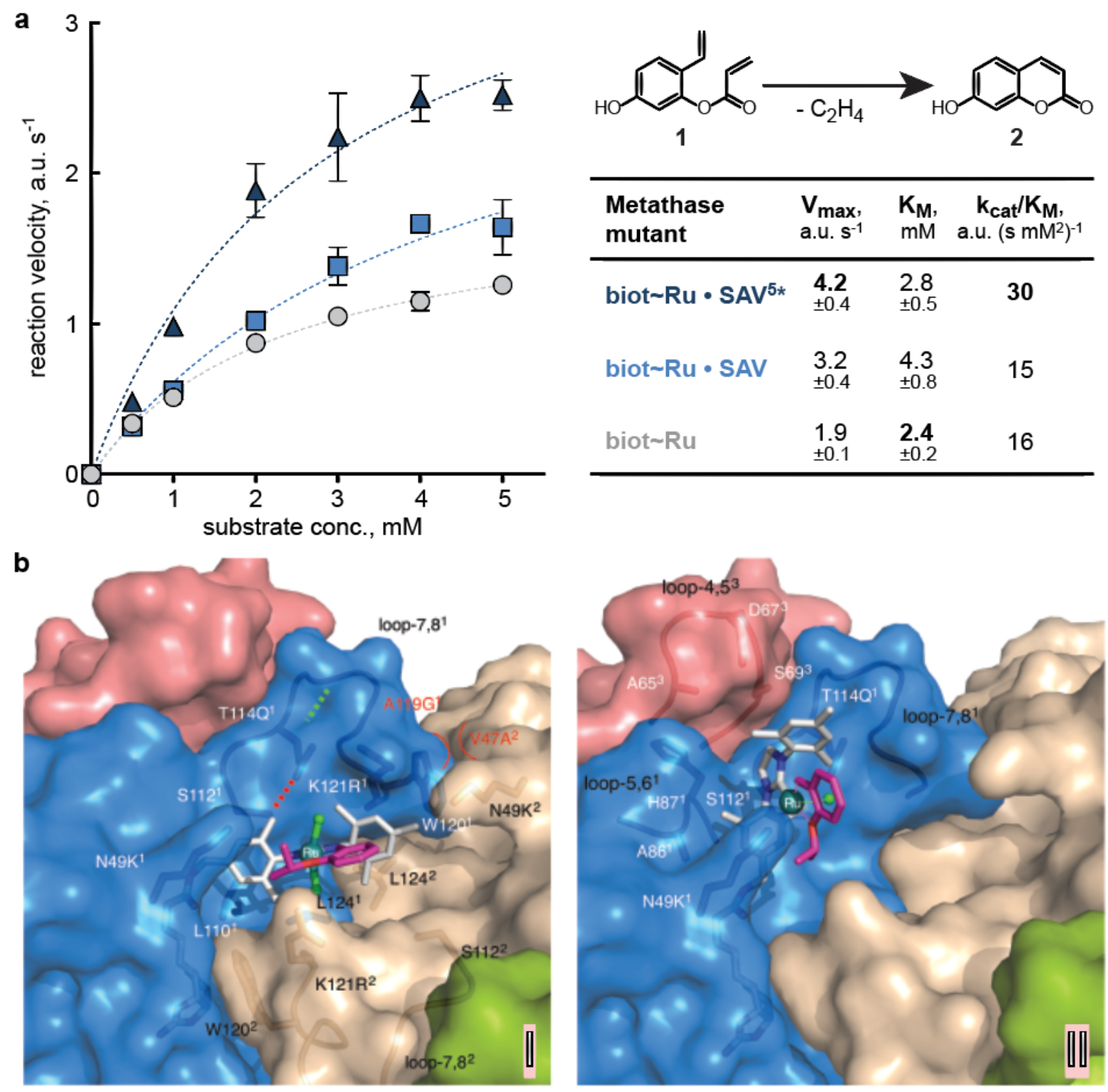

Figure 3 | Characterization of artificial metathases. a, Michaelis-Menten kinetics of free biot $\sim \mathbf{R u}$ (circles), biot $\sim \mathbf{R u} \cdot \mathrm{SAV}$ (squares) and biot $\sim \mathbf{R u} \cdot \mathrm{SAV}^{5 *}$ (triangles) were performed relying on umbelliferone 2 formation by metathesis. Symbols represent mean values for three kinetic in vitro experiments with s.d.. Dashed lines represent the best fit $\left(R^{2}=0.99, R^{2}=0.98\right.$, $\left.R^{2}=0.97\right)$ to a Michaelis-Menten model with parameters maximum velocity $\left(V_{\max }\right)$, substrate affinity $\left(K_{M}\right)$ as summarized (box) together with catalytic efficiency $\left(\mathrm{K}_{\text {cat }} / \mathrm{K}_{M}\right)$. b, X-Ray structure (PDB: 5F2B) of the evolved metathase biot Ru $\cdot S^{2} V^{5 *}$ in the two active site conformations I and II. Biot Ru and close protein residues are depicted in stick model. Flexibility of loop-7, $8^{1}$ is increased by $\mathrm{H}$-bond swapping with mutation $\mathrm{T}_{114 \mathrm{Q}^{1}}$ (red for green dashed line), entropy-raising mutation $A 119 G^{1}$ and bulk-reducing $V 47 A^{2}$. The unresolved alkylidene ligand (magenta) in the crystal structure was modeled. 

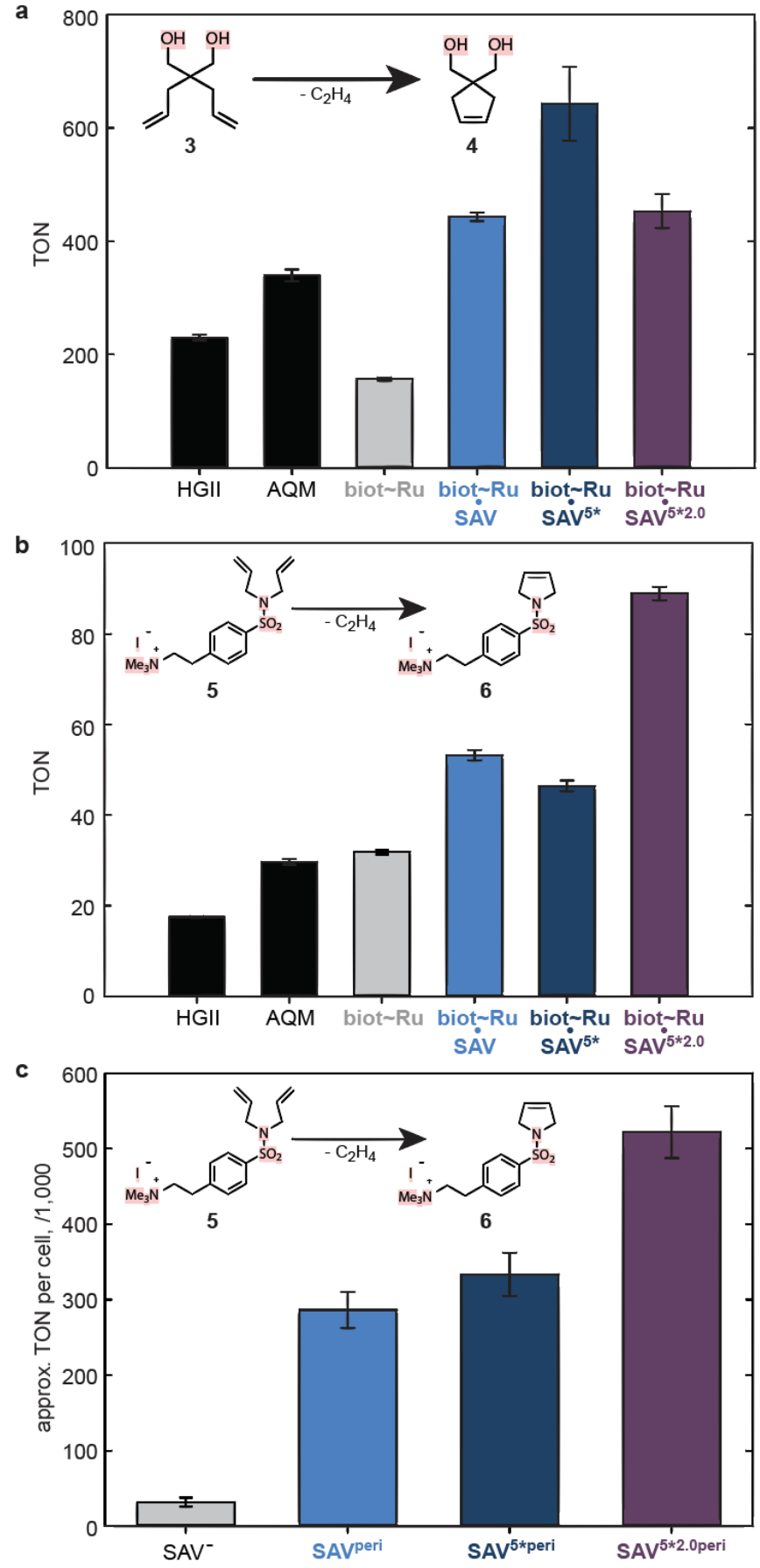

Figure 4 | Substrate-specific evolution of an artificial metathase. The performance of the purified artificial metathase was evaluated in vitro for two water soluble substrates: the diallyl propanediol 3 (a) as well as the cationic diallyl-sulfonamide 5 (b). Randomization of residue $\mathrm{R} 121$ in $\mathrm{SAV}^{5 *}$ and subsequent periplasmic screening led to the identification of the quintuple mutant $\mathrm{SAV}^{5 * 2.0}$ (47A-49K-114Q-119G-R121L) with significantly enhanced activity for the cationic substrate 5 both in vitro using purified protein (b) as well as in vivo (c). Bars represent mean turnover number (TON) of three replicate in vitro experiments $(\mathbf{a}, \mathbf{b})$ or average TON per cell detected in eight independent replicate cultures (c) with s.d. (error bars). For comparison, the performance of two commercially available metathesis catalysts 
$\left(2^{\text {nd }}\right.$ generation Hoveyda-Grubbs (HGII) and AquaMet (AQM), see Extended Data Fig. 6) is depicted. 